Investigaciones Fenomenológicas, vol. Monográfico 4/I (2013): Razón y vida, 15-20. e-ISSN: $1885-1088$

\title{
GALERÍA DE FOTOS
}

\section{Photo Gallery}

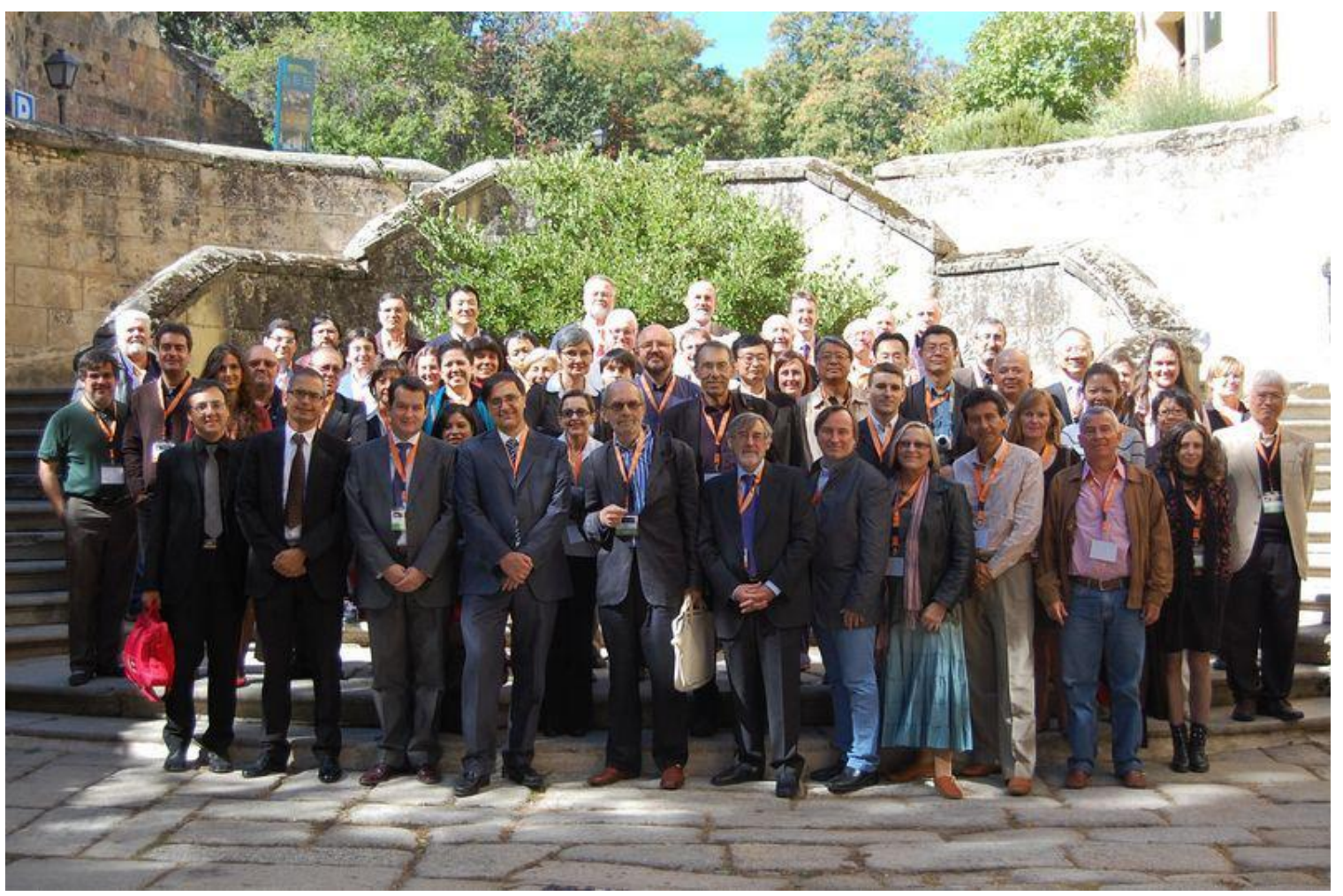

Asistentes al IV Congreso OPO tras la ceremonia de inauguración.

Assistants to the IV Congress OPO after the opening ceremony. 
Lunes, 19 Septiembre 2011

September 19, 2011 (Monday)

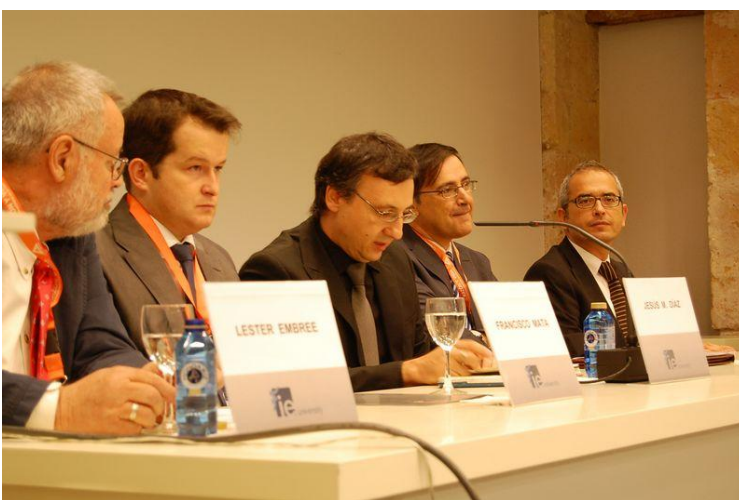

Imagen 1. De izquierda a derecha: Lester Embree, Francisco Mata, Jesús M. Díaz, Agustín Serrano y Antonio López.

Imagen 1. From left to right: Lester Embree, Francisco Mata, Jesús M. Díaz, Agustín Serrano and Antonio López.

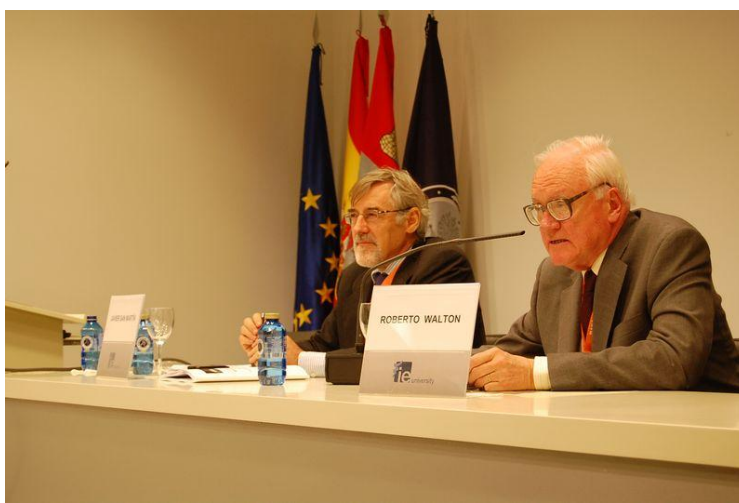

Imagen 3. De izquierda a derecha: Javier San Martín y Roberto Walton.

Imagen 3. From left to right: Javier San Martín and Roberto Walton.

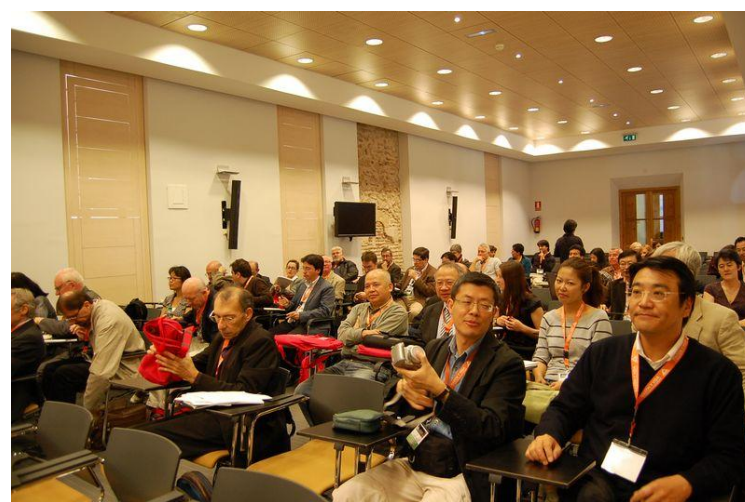

Imagen 2. Asistentes al IV Congress OPO en la inauguración del congreso.

Imagen 2. Assistants to the IV Congress $\mathrm{OPO}$ at the meeting opening.

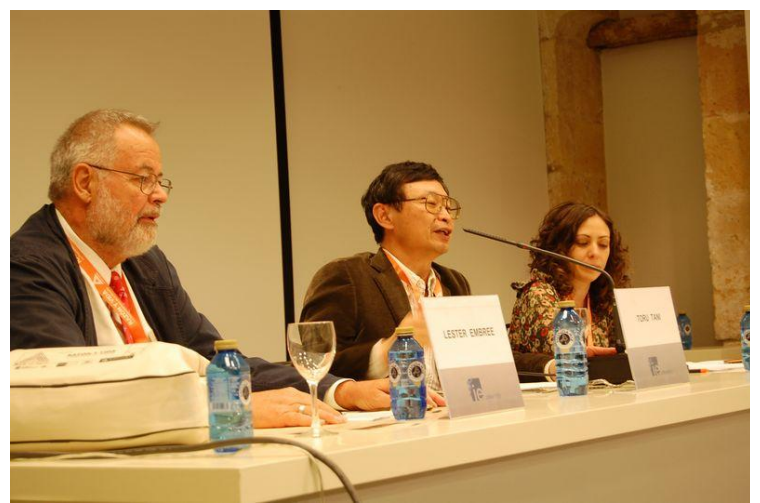

Imagen 4. De izquierda a derecha: Lester Embree, Toru Tani y Daniela Griselda López.

Imagen 4. From left to right: Lester Embree, Toru Tani and Daniela Griselda López. 
Martes, 20 Septiembre 2011

September 20, 2011 (Tuesday)

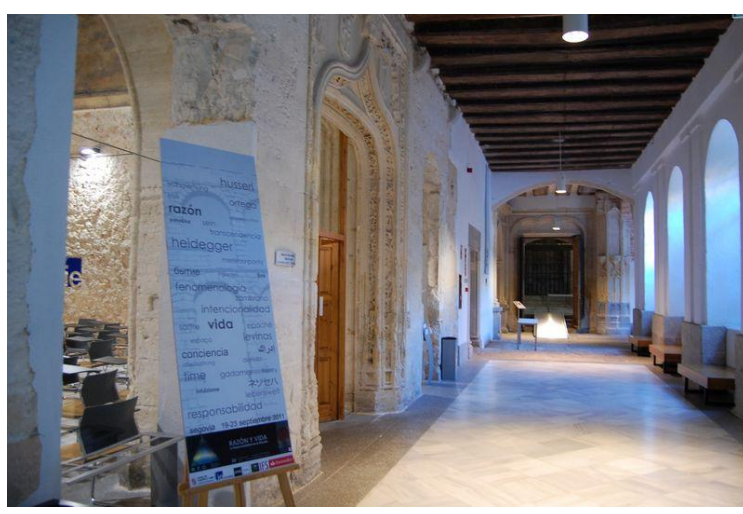

Imagen 5. Claustro en el IE University, Segovia.

Imagen 5. Cloister at IE University, Segovia.

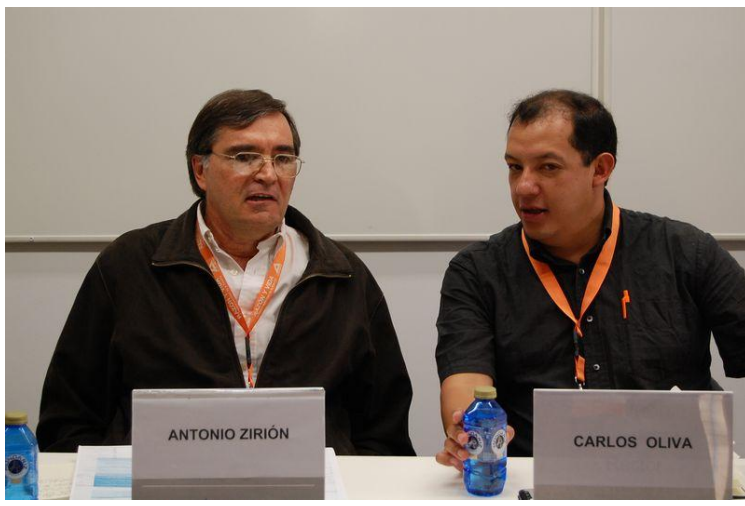

Imagen 7. De izquierda a derecha: Antonio Zirión y Carlos Oliva.

Imagen 7. From left to right: Antonio Zirión and Carlos Oliva.

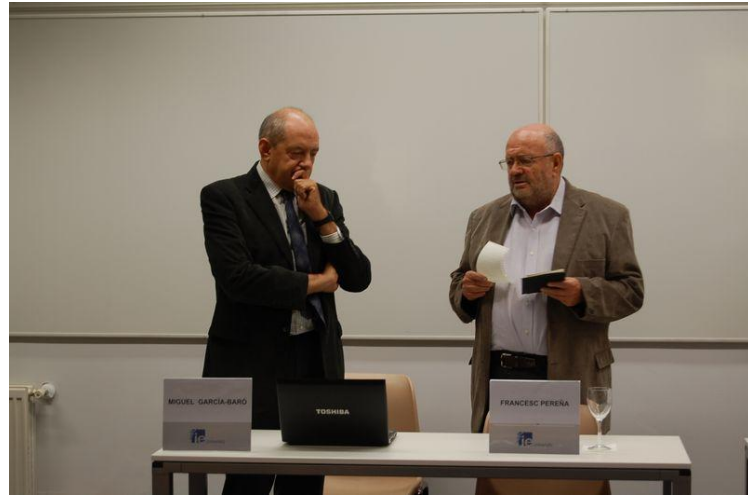

Imagen 6. De izquierda a derecha: Miguel García-Baro y Francesc Pereña.

Imagen 6. From left to right: Miguel GarcíaBaro and Francesc Pereña

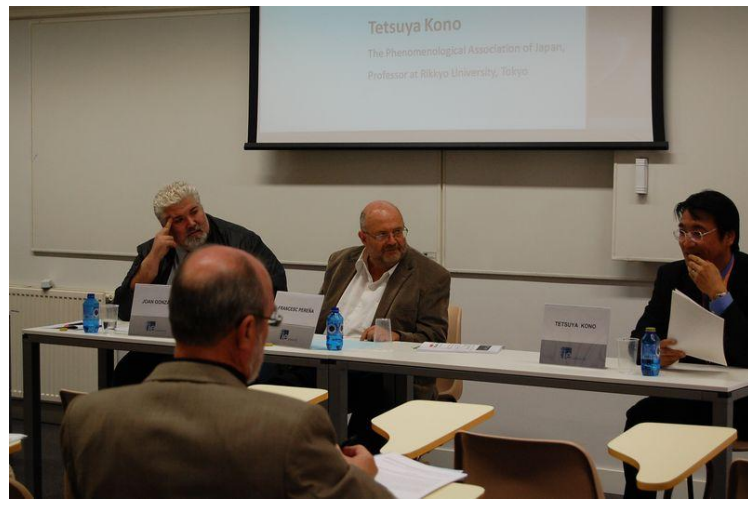

Imagen 8. De izquierda a derecha: Joan González, Francesc Pereña y Tetsuya Kono.

Imagen 8. From left to right: Joan González, Francesc Pereña and Tetsuya Kono. 
Miércoles, 21 Septiembre 2011

September 21, 2011 (Wednesday)

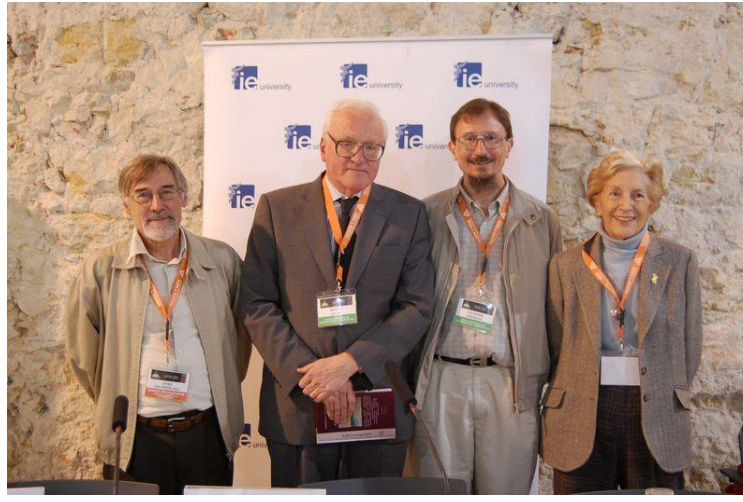

Imagen 9. De izquierda a derecha: Javier San Martín, Roberto Walton, Luis Román Rabanaque y Julia V. Iribarne.

Imagen 9. From left to right: Javier San Martín, Roberto Walton, Luis Román Rabanaque and Julia V. Iribarne.

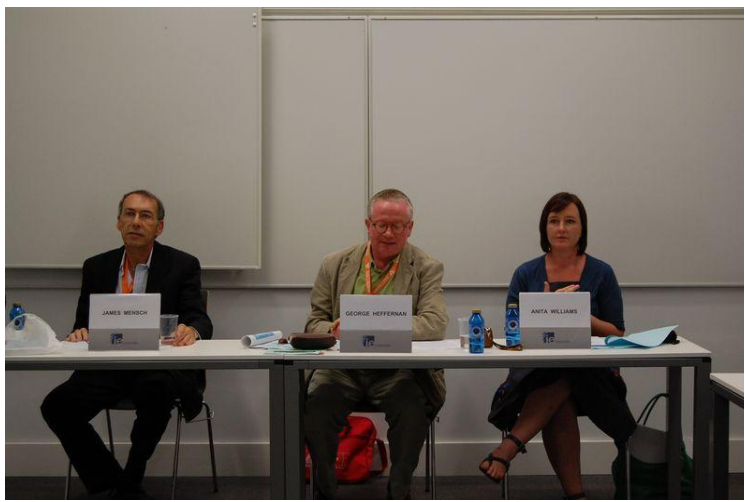

Imagen 11. De izquierda a derecha: James Mensch, George Heffernan y Anita Williams.

Imagen 11. From left to right: James Mensch, George Heffernan and Anita Williams.

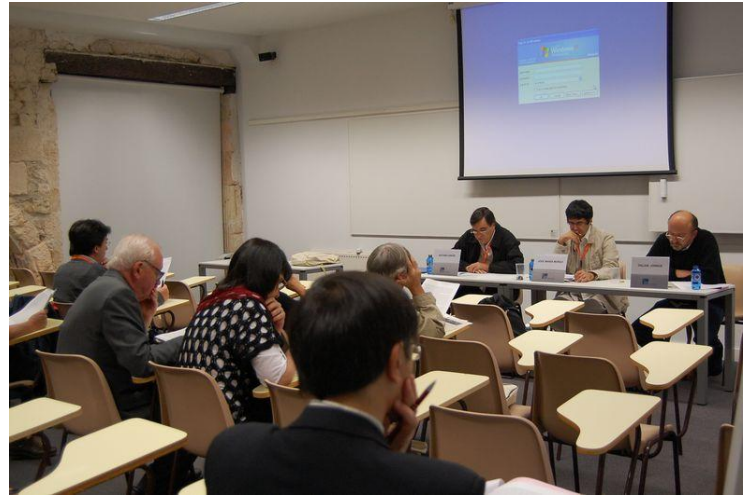

Imagen 10. Visión general de una sesión. En la mesa (de izquierda a derecha): Antonio Zirión, Jose María Muñoz y Dalius Jonkus.

Imagen 10. Overview of a session. At the table (from left to right) Antonio Zirión, Jose María Muñoz and Dalius Jonkus.

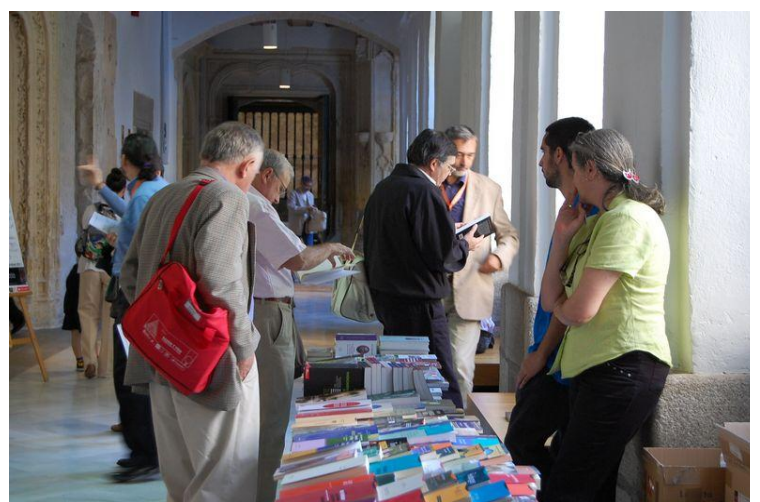

Imagen 12. Exposición libros en el claustro del IE University.

Imagen 12. Exposure books in the cloister of IE University. 
Jueves, 22 Septiembre 2011

September 22, 2011 (Thursday)
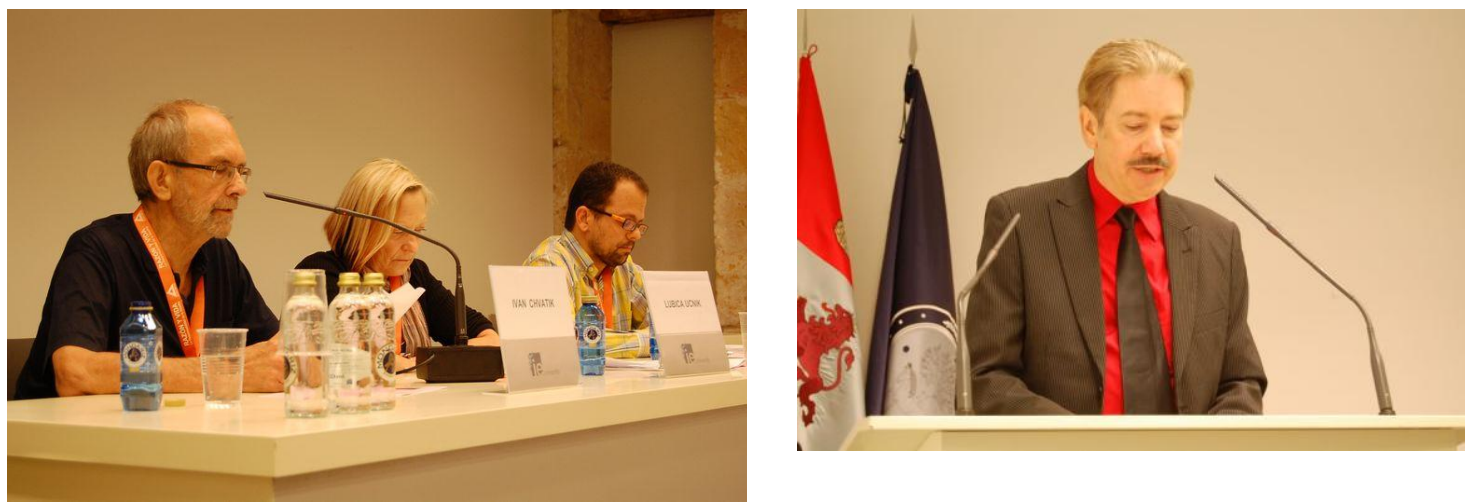

Imagen 13. De izquierda a derecha: Ivan Imagen 14. Hans Rainer Sepp. Chvatik, L'ubica Učník y Ivan Ortega.

Imagen 13. From left to right: Ivan Chvatik, Imagen 14. Hans Rainer Sepp. L'ubica Učník and Ivan Ortega.



Imagen 15. Asistente al IV Congreso OPO en la conferencia de Rainer Seep.

Imagen 15. Assistants to the IV Congress $O P O$ at Rainer Seep's conference.

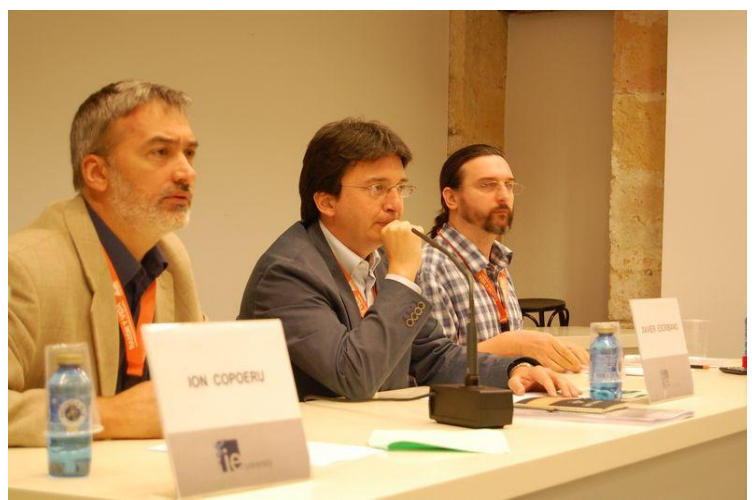

Imagen 16. De izquierda a derecha: Ion Copoeru, Xavier Escribano y Cristian Ciocan.

Imagen 16. From left to right: Ion Copoeru, Xavier Escribano and Cristian Ciocan. 
Viernes, 23 Septiembre 2011

September 23, 2011 (Friday)

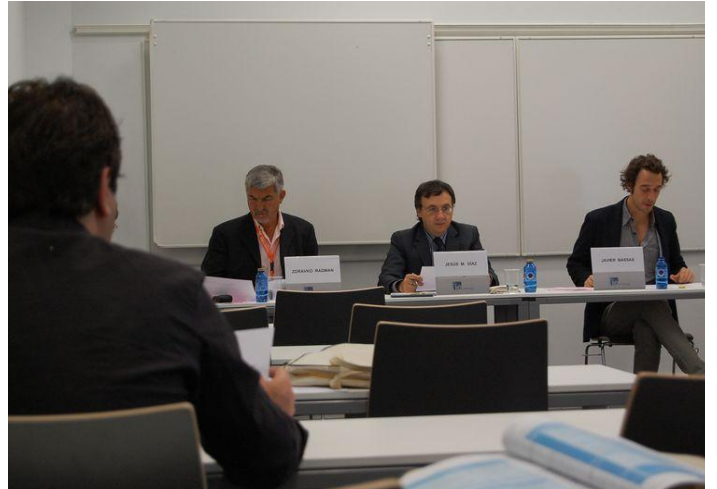

Imagen 17. De izquierda a derecha: Zdravko Radman, Jesús M. Díaz y Xavier Bassas.

Imagen 17. From left to right: Zdravko Radman, Jesús M. Díaz and Xavier Bassas.



Imagen 19. De izquierda a derecha: Thomas Nenon y Ion Copoeru.

Imagen 19. From left to right: Thomas Nenon and Ion Copoeru.

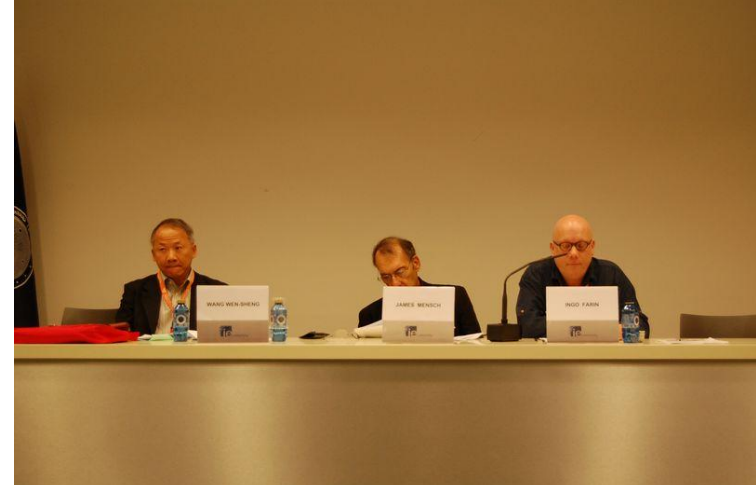

Imagen 18. De izquierda a derecha: Wang Wen-Sheing, James Mensch y Ingo Farin.

Imagen 18. From left to right: Wang WenSheing, James Mensch and Ingo Farin.

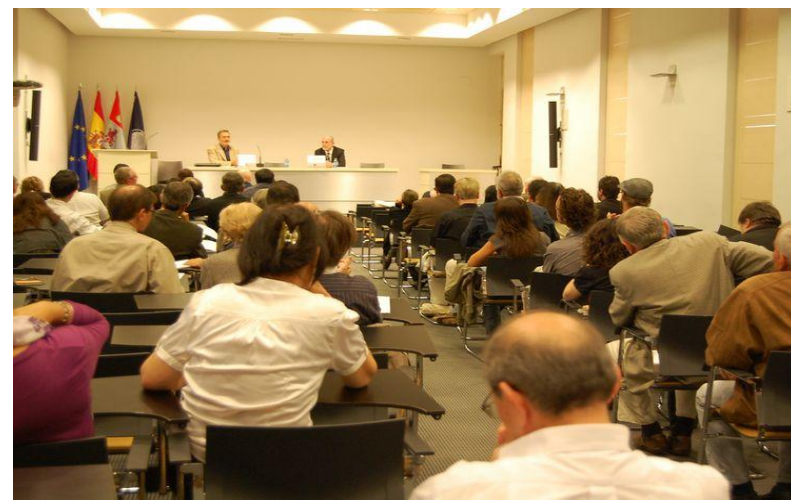

Imagen 20. Participantes asistiendo a la conferencia de Thomas Nenon.

Imagen 20. Participants attending Thomas Nenon's conference. 